\section{Comparação da mortalidade neonatal em recém-nascidos de muito baixo peso ao nascimento, em maternidades do Município do Rio de Janeiro, Brasil}

\author{
Comparison of neonatal mortality in very \\ low birth weight newborns at maternity \\ hospitals in the city of Rio de Janeiro, Brazil
}

\author{
${ }^{1}$ Faculdade de Ciências \\ Médicas, Universidade \\ do Estado do Rio de Janeiro, \\ Rio de Janeiro, Brasil. \\ 2 Instituto de Medicina \\ Social, Universidade \\ do Estado do Rio de Janeiro, \\ Rio de Janeiro, Brasil. \\ Correspondência \\ J. L. M. B. Duarte \\ Faculdade de Ciências \\ Médicas, Universidade \\ do Estado do Rio de Janeiro. \\ Rua Nelson Rodrigues 162, \\ Rio de Janeiro, $R J$ \\ 22793-260, Brasil. \\ jlduarte@centroin.com.br
}

\begin{abstract}
This study was a comparison of neonatal mortality rates in four maternity hospitals in the city of Rio de Janeiro, Brazil. The study population consisted of newborns with birth weight below 1,500g. The research instrument was a questionnaire with data reported by the mother and collected from the patient record. For each maternity hospital the standardized mortality ratio (SMR) was calculated using the direct and indirect method, using the weight distribution of the National Institute of Child Health and Human Development Neonatal Research as the standard. The final sample consisted of 487 newborns. Standardizations by the direct and indirect method showed high mortality rates in all four institutions; those showing the largest number of newborns in the lowest weight brackets were those with the lowest SMR values. The lowest mortality ratio by weight bracket was in the 500 to $749 \mathrm{~g}$ group. The SMR results were inversely associated with the quantitative population of newborns in the lowest weight brackets. The mortality coefficients showed high rates, especially in the highest weight brackets. The results indicate deficient quality of perinatal care.

Neonatal Mortality; Very Low Birth Weight Infant; Maternity Hospitals
\end{abstract}

José Luiz Muniz Bandeira Duarte 1 Gulnar Azevedo Silva Mendonça ${ }^{2}$

\section{Introdução}

As taxas de mortalidade neonatal são os indicadores mais utilizados para avaliação de qualidade no atendimento neonatal. A comparação de resultados dessas taxas entre os serviços de neonatologia pode auxiliar no planejamento de ações que visem à melhoria do atendimento aos recém-nascidos.

Os estudos realizados para avaliação da atenção neonatal devem considerar que a distribuição do peso ao nascimento pode ser bem variada entre diferentes serviços e isso deve ser sempre considerado em estudos que comparam o risco de óbito em diferentes instituições. No intuito de melhor lidar com esse problema, Mallet \& Knoss 1, em 1970, utilizaram o método de padronização por peso ao nascimento como forma de avaliar os demais fatores associados independentemente do risco inerente ao peso. Wilcox \& Russel 2, em 1983, observaram em estudo no qual usaram a padronização direta, que uma população de recém-nascidos comparada a uma outra, apresenta uma diferença na média de peso ao nascimento 15,0 a $25,0 \%$ maior do que os resultados encontrados, esse fato favoreceu as populações com as maiores médias de pesos ao nascimento.

Barros et al. 3 , cerca de vinte anos atrás, referiram que em países em desenvolvimento, como o Brasil, os estudos de saúde perinatal são escassos; quando existentes, costumam basear- 
se na avaliação de amostras não representativas da população e as taxas de mortalidade costumam ser calculadas com base em atestados de óbitos, subestimando a gravidade da situação. Os autores compararam as taxas de mortalidade perinatal na cidade de Pelotas, no Sul do Brasil, com as da Suécia, e a partir da padronização direta e indireta concluíram que a assistência à gestante e ao recém-nascido contribuiu para o aumento de óbitos, mais do que o risco de nascer com baixo peso.

O recém-nascido de muito baixo peso ao nascimento, aquele com peso ao nascimento inferior a $1.500 \mathrm{~g}$, representa a metade do percentual de morte na taxa de mortalidade infantil dos Estados Unidos 4. No Município do Rio de Janeiro essa mesma taxa é responsável, do total de óbitos, por 51,6\% (Gerência de Informações Epidemiológicas, Secretaria Municipal de Saúde do Rio de Janeiro. Taxa de Mortalidade Neonatal Peso-específica do Rio de Janeiro, 1996 a 2002. http://www.saude.rio.rj.gov.br, acessado em 03/Out/2004), tornando esse grupo de recém-nascido o foco de atenção em estudos que visem ações para diminuição da mortalidade infantil.

Nos últimos anos, vários estudos identificaram fatores associados ao risco de morte entre os recém-nascidos. Fatores como: visitas ao prénatal 3,4,5, uso de corticosteróide neonatal 6 , surfactante pulmonar 7 e nutrição parenteral 8 são protetores de óbito neonatal. Enquanto o sexo masculino 9, CRIB 10 (Clinical Risk Index Baby - índice de gravidade) alto, extremo baixo peso ao nascer 11 e uso de ventilação mecânica ${ }^{9}$ mostraram associação com o risco de morte.

Este estudo tem como objetivo a comparação das taxas de mortalidade neonatal em quatro maternidades do Município do Rio de Janeiro por meio da padronização por peso ao nascer segundo método direto e indireto.

\section{Material e métodos}

A população do estudo foi recrutada entre todos os recém-nascidos com peso, ao nascimento, superior a $500 \mathrm{~g}$ e igual/inferior a $1.500 \mathrm{~g}$, em quatro maternidades localizadas no Município do Rio de Janeiro. As maternidades eram consideradas como centros terciários na assistência neonatal na cidade do Rio de Janeiro. Todos os centros apresentaram predomínio de recémnascidos da própria maternidade. O estudo compreendeu o período entre 28 de agosto de 2001 a 28 de setembro de 2002 , e todos os recém-nascidos incluídos foram acompanhados

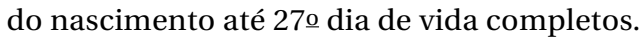

Foram excluídos os recém-nascidos com idade gestacional inferior a 22 semanas ou portadores de malformações congênitas incompatíveis com a vida, como a anencefalia, a trissomia dos 18, a agenesia renal, bem como as crianças transferidas para instituição não participante do estudo.

\section{Coleta de dados}

O peso ao nascimento foi aferido na sala de parto ou na admissão do recém-nascido no berçário ou na unidade de terapia intensiva e estratificada com intervalos de $250 \mathrm{~g}$, além disso, foram coletados dados do prontuário em relação ao recém-nascido. A coleta de dados foi realizada por um médico em cada instituição e supervisionado semanalmente pelo pesquisador de campo.

\section{Análise dos dados}

Inicialmente foi realizada a descrição dos dados levantados por maternidade, que incluiu a distribuição dos recém-nascidos vivos, dos recém-nascidos de muito baixo peso e do percentual de óbitos em cada instituição.

Em seguida foram calculadas, para cada instituição, as Razões Padronizadas de Mortalidade (RPM) pelo peso ao nascimento com o método direto e tendo como padrão a distribuição por peso ao nascimento dos Estados Unidos, disponível no banco de dados do National Institute of Child Health and Human Development Neonatal Research 12. Foram ainda calculadas as RPM ajustadas por peso ao nascimento utilizando-se o método indireto, tendo como referência os coeficientes de mortalidade do mesmo banco 12 por faixa de peso.

O cálculo da RPM foi realizado para cada instituição tendo como padrão a distribuição populacional do banco de dados do National Institute of Child Health and Human Development Neonatal Research 12 . O cálculo é aferido por meio da razão do número de óbitos observados sobre o esperado e multiplicado por 100 . $\mathrm{RPM}=(\mathrm{O} / \mathrm{E}) \times 100$

Por último, utilizando-se os coeficientes das instituições estudadas e os dos Estados Unidos para cada faixa de peso ao nascimento, foi calculada a variação percentual entre os óbitos observados sobre os esperados.

Os dados coletados foram armazenados no programa Stata, versão 6.0, e Epi Info versão 6.04 . 


\section{Resultados}

Foram incluídos no estudo um total de 509 recém-nascidos de muito baixo peso. Desses, foram excluídos 17 (3,3\%) recém-nascidos transferidos para outras instituições e cinco com malformações. A amostra final contou assim com 487 recém-nascidos $(96,0 \%)$ da amostra inicialmente planejada.

A Tabela 1 mostra o total de nascimentos por maternidade e o respectivo percentual de recém-nascidos de muito baixo peso e óbitos. As instituições com os mais baixos percentuais de recém-nascidos vivos foram aquelas que apresentaram os maiores percentuais de nascimento de recém-nascidos de muito baixo peso, devido a um direcionamento do atendimento à gestação de alto risco. As outras duas maternidades eram direcionadas a um atendimento universal, mas possuíam unidades de tratamento de recém-nascidos de alto risco. A Instituição 1 apresentou o menor número de recém-nascidos vivos, o maior percentual de recém-nascidos de muito baixo peso e a menor média de peso ao nascimento. A Instituição 4 mostrou o maior número de recém-nascidos vivos, o menor percentual de recém-nascidos de muito baixo peso e a maior média de peso. Embora a Instituição 3 não fosse a que apresentou o maior percentual de recém-nascidos de muito baixo peso, foi a que mostrou o maior percentual de óbitos, e a Instituição 2, o menor. A diferença entre a média de peso mais baixa e a mais elevada foi de $10,6 \%$.

A Tabela 2 mostra a distribuição dos recémnascidos de muito baixo peso em cada instituição estudada, e observamos que as instituições 1 e 3 foram as que apresentaram o maior percentual de recém-nascidos nas faixas inferiores de pesos ao nascimento e os maiores percentuais de mortes, mostrados na Tabela 1.

A Tabela 3 apresenta a distribuição de óbitos entre recém-nascidos de muito baixo peso observados e esperados por meio da padronização direta, utilizando como população da base de dados a encontrada no estudo do National Institute of Child Health and Human Development Neonatal Research. As taxas de mortalidade se mostraram bastante elevadas em todas as instituições estudadas. As instituições 1 e 3 por apresentarem o maior percentual de recém-nascidos nas faixas com os pesos ao nas-

Tabela 1

Total de nascimentos, número de recém-nascidos e muito baixo peso, e porcentagem de óbitos por instituição.

\begin{tabular}{|c|c|c|c|c|c|c|}
\hline \multirow[t]{3}{*}{ Instituição } & \multirow{3}{*}{$\begin{array}{c}\text { Recém-nascidos } \\
\text { vivos } \\
N\end{array}$} & \multicolumn{5}{|c|}{ Recém-nascidos de muito baixo peso } \\
\hline & & \multicolumn{3}{|c|}{ Total } & \multicolumn{2}{|c|}{ Óbitos } \\
\hline & & $n$ & $\%$ & Peso médio (g) & $\mathrm{n}$ & $\%$ \\
\hline 1 & 667 & 42 & 6,7 & $1.023,3$ & 11 & 26,0 \\
\hline 2 & 990 & 59 & 6,0 & $1.081,1$ & 6 & 10,0 \\
\hline 3 & 4.216 & 196 & 4,6 & $1.087,3$ & 72 & 37,0 \\
\hline 4 & 5.393 & 190 & 3,8 & $1.132,2$ & 37 & 19,0 \\
\hline Total & 11.266 & 487 & 4,2 & $1.080,9$ & 126 & 26,0 \\
\hline
\end{tabular}

Tabela 2

Distribuição dos recém-nascidos de muito baixo peso em cada instituição estudada.

\begin{tabular}{|c|c|c|c|c|c|c|c|c|c|c|}
\hline \multirow{2}{*}{$\begin{array}{l}\text { Peso ao } \\
\text { nascer }(\mathrm{g})\end{array}$} & \multicolumn{10}{|c|}{ Instituição } \\
\hline & $n$ & $\%$ & $\mathrm{n}$ & $\%$ & $\mathrm{n}$ & $\%$ & $\mathrm{n}$ & $\%$ & $\mathrm{n}$ & $\%$ \\
\hline $500-749$ & 7 & 16,67 & 5 & 8,47 & 30 & 15,31 & 16 & 8,42 & 58 & 11,91 \\
\hline $750-999$ & 14 & 33,33 & 15 & 25,42 & 41 & 20,92 & 45 & 23,68 & 115 & 23,61 \\
\hline $1.000-1.249$ & 9 & 21,43 & 21 & 35,59 & 54 & 27,55 & 54 & 28,42 & 138 & 28,34 \\
\hline $1.250-1.500$ & 12 & 28,57 & 18 & 30,51 & 71 & 36,22 & 75 & 39,47 & 176 & 36,14 \\
\hline
\end{tabular}


Óbitos observados e esperados e os coeficientes padronizados pelo método direto e peso ao nascer.

\begin{tabular}{|c|c|c|c|c|c|c|c|c|c|c|c|c|c|}
\hline \multirow{3}{*}{$\begin{array}{l}\text { Peso ao } \\
\text { nascer }(\mathrm{g})\end{array}$} & \multirow{3}{*}{$\begin{array}{c}\text { Estados } \\
\text { Unidos* } \\
\text { Nascidos } \\
\text { vivos } \\
n\end{array}$} & \multicolumn{3}{|c|}{ Instituição 1} & \multicolumn{3}{|c|}{ Instituição 2} & \multicolumn{3}{|c|}{ Instituição 3} & \multicolumn{3}{|c|}{ Instituição 4} \\
\hline & & \multirow[t]{2}{*}{$\mathrm{CMB}$} & \multicolumn{2}{|c|}{ Óbitos (n) } & \multirow[t]{2}{*}{$\mathrm{CMB}$} & \multicolumn{2}{|c|}{ Óbitos (n) } & \multirow[t]{2}{*}{ CMB } & \multicolumn{2}{|c|}{ Óbitos (n) } & \multirow[t]{2}{*}{$\mathrm{CMB}$} & \multicolumn{2}{|c|}{ Óbitos (n) } \\
\hline & & & $\begin{array}{l}\text { Obser- } \\
\text { vado }\end{array}$ & $\begin{array}{l}\text { Espe- } \\
\text { rado }\end{array}$ & & $\begin{array}{l}\text { Obser- } \\
\text { vado }\end{array}$ & $\begin{array}{l}\text { Espe- } \\
\text { rado }\end{array}$ & & $\begin{array}{l}\text { Obser- } \\
\text { vado }\end{array}$ & $\begin{array}{l}\text { Espe- } \\
\text { rado }\end{array}$ & & $\begin{array}{l}\text { Obser- } \\
\text { vado }\end{array}$ & $\begin{array}{l}\text { Espe- } \\
\text { rado }\end{array}$ \\
\hline $500-749$ & 1.002 & 714 & 5 & 7 & 400 & 2 & 5 & 900 & 27 & 40 & 688 & 11 & 30 \\
\hline $750-999$ & 1.084 & 286 & 4 & 3 & 133 & 2 & 2 & 414 & 17 & 20 & 311 & 14 & 15 \\
\hline $1.000-1.249$ & 1.053 & 111 & 1 & 1 & 48 & 1 & 1 & 333 & 18 & 16 & 148 & 8 & 7 \\
\hline $1.250-1.500$ & 1.299 & 83 & 1 & 1 & 56 & 1 & 1 & 141 & 10 & 8 & 53 & 4 & 3 \\
\hline Total & 4.438 & & 11 & 12 & & 6 & 9 & & 72 & 84 & & 37 & 55 \\
\hline Variação (\%) & & \multicolumn{3}{|c|}{$+9,1$} & \multicolumn{3}{|c|}{$+50,0$} & \multicolumn{3}{|c|}{$+16,6$} & \multicolumn{3}{|r|}{$+48,6$} \\
\hline RPM & & \multicolumn{3}{|c|}{91,7} & \multicolumn{3}{|c|}{66,7} & \multicolumn{3}{|c|}{85,7} & & & 67,3 \\
\hline
\end{tabular}

* National Institute of Child Health and Human Development Neonatal Research.

$\mathrm{CMB}=$ coeficiente de mortalidade bruto por mil recém-nascidos vivos; RPM = razão padronizada de mortalidade.

cimento mais baixos, foram aquelas que mostraram os maiores valores de RPM.

Na Tabela 4 os resultados mostraram elevadas taxas de mortalidade em todas as instituições estudadas, caso utilizássemos as taxas de mortalidade do National Institute of Child Health and Human Development Neonatal Research como padrão. A Instituição 3 foi a que apresentou a taxa mais elevada $(\mathrm{RPM}=338,0)$, seguida da Instituição 4 (RPM = 225,6), depois da Instituição $1(\mathrm{RPM}=150,7)$ e por último a Instituição $2(\mathrm{RPM}=114,3)$. Os resultados mostraram trocas de posições, observadas na Tabela 1, entre a Instituição 1 (terceiro maior percentual de óbito) e a 4 (segundo maior percentual de óbito) quando calculada a RPM. As instituições com os menores percentuais de mortes nas faixas de pesos mais baixos foram aquelas que apresentaram os menores valores de RPM.

A razão de mortalidade por faixa de peso ao nascimento para cada instituição, comparando-se as taxas observadas com as taxas de mortalidade do National Institute of Child Health and Human Development Neonatal Research, foi menor para a faixa de peso ao nascimento entre 500 e $749 \mathrm{~g}$. Os maiores valores foram encontrados entre as maiores faixas de peso ao nascimento (Tabela 5). A Instituição 2 mostrou resultados bastante animadores com valores semelhantes, na menor faixa de peso, e até melhores, na faixa de peso entre $1.000 \mathrm{~g}$ e $1.249 \mathrm{~g}$, quando comparados com o National Institute of Child Health and Human Development Neonatal Research. A Instituição 3 mostrou razões elevadas em todas as faixas de peso estudadas.

\section{Discussão}

O cálculo das RPM por peso ao nascimento pelo método direto mostrou um aumento da mortalidade neonatal em todas as instituições estudadas, caso a distribuição populacional fosse do banco de dados dos Estados Unidos. As instituições 1 e 3 foram aquelas que apresentaram os maiores valores. Os resultados descritos por Wilcox \& Russel 2, que mostraram valores mais elevados entre as populações que apresentavam as maiores médias de peso, não foram observados no nosso estudo porque a variação percentual de peso ao nascimento entre as instituições estudadas (10,6\%) não ultrapassou o valor, que o estudo desses autores, mostrou ser importante (maior que 15,0 a 25,0\%). Esses mesmos autores em outro estudo 13 mostraram que a população que apresentasse a maior quantidade de recém-nascidos na faixa de menor peso ao nascimento, evidência da distribuição residual da curva gaussiana do peso ao nascimento, seria a população com os piores resultados quando se calculasse a RPM. As instituições 1 e 3 mostraram exatamente esse problema, foram elas que apresentaram os maiores percentuais de recém-nascidos nas faixas de menores pesos. Essa mesma observação foi feita por Guyer et al. 14, quando compararam a baixa mortalidade neonatal na Suécia com a dos Estados Unidos. Os autores, utilizando a padronização direta por peso ao nascimento, concluíram que, a Suécia, apresentava uma pequena quantidade de recém-nascidos de muito baixo peso, produzindo coeficiente de mortalidade baixo. A explicação para essa ocorrência seria a de que nessas faixas de me- 
Óbitos observados e esperados e os coeficientes padronizados pelo método indireto e peso ao nascer.

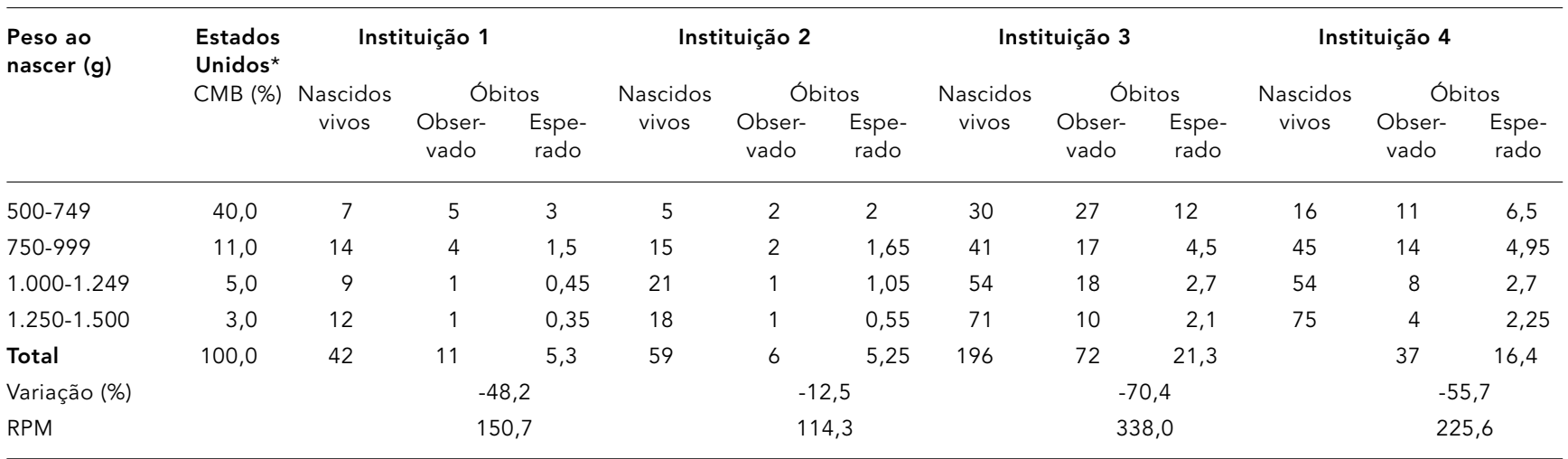

* National Institute of Child Health and Human Development Neonatal Research.

$\mathrm{CMB}=$ coeficiente de mortalidade bruto por mil recém-nascidos vivos; RPM = razão padronizada de mortalidade.

Razão de mortalidade por faixa de peso para cada Instituição comparada aos dados dos Estados Unidos.

\begin{tabular}{|c|c|c|c|c|c|c|c|c|c|c|}
\hline \multirow{2}{*}{$\begin{array}{l}\text { Peso ao } \\
\text { nascer (g) }\end{array}$} & \multicolumn{2}{|c|}{ Estados Unidos* } & \multicolumn{2}{|c|}{ Instituição 1} & \multicolumn{2}{|c|}{ Instituição 2} & \multicolumn{2}{|c|}{ Instituição 3} & \multicolumn{2}{|c|}{ Instituição 4} \\
\hline & $\begin{array}{c}\text { Nascidos } \\
\text { vivos } \\
n\end{array}$ & $\mathrm{CMB}$ & $\begin{array}{c}\text { Coeficiente } \\
\text { de mor- } \\
\text { talidade } \\
\%\end{array}$ & $\begin{array}{c}\text { Razão } \\
\text { instituição/ } \\
\text { Estados } \\
\text { Unidos** }\end{array}$ & $\begin{array}{c}\text { Coeficiente } \\
\text { de mor- } \\
\text { talidade } \\
\%\end{array}$ & $\begin{array}{c}\text { Razão } \\
\text { instituição/ } \\
\text { Estados } \\
\text { Unidos }\end{array}$ & $\begin{array}{c}\text { Coeficiente } \\
\text { de mor- } \\
\text { talidade } \\
\%\end{array}$ & $\begin{array}{c}\text { Razão } \\
\text { instituição/ } \\
\text { Estados } \\
\text { Unidos }\end{array}$ & $\begin{array}{c}\text { Coeficiente } \\
\text { de mor- } \\
\text { talidade } \\
\% \text { \% }\end{array}$ & $\begin{array}{l}\text { Razão } \\
\text { instituição/ } \\
\text { Estados } \\
\text { Unidos }\end{array}$ \\
\hline $500-749$ & 1.002 & 400 & 714 & 1,8 & 400 & 1,0 & 900 & 2,3 & 688 & 1,7 \\
\hline 750-999 & 1.084 & 110 & 286 & 2,6 & 133 & 1,2 & 414 & 3,8 & 311 & 2,8 \\
\hline $1.000-1.249$ & 1.053 & 50 & 111 & 2,2 & 48 & 0,9 & 333 & 6,7 & 148 & 3,0 \\
\hline $1.250-1.500$ & 1.299 & 30 & 83 & 2,8 & 56 & 1,9 & 141 & 4,7 & 53 & 1,8 \\
\hline
\end{tabular}

* National Institute of Child Health and Human Development Neonatal Research.

** Relação do coeficiente de mortalidade de cada instituição/coeficiente de mortalidade do National Institute of Child Health and Human Development Neonatal Research.

$\mathrm{CMB}=$ coeficiente de mortalidade bruto por mil recém-nascidos vivos.

nores pesos é justamente onde ocorreria a maior mortalidade entre os recém-nascidos.

O estudo fez a comparação com base na população do banco de dados do National Institute of Child Health and Human Development Neonatal Research, este com um percentual bem maior de recém-nascidos na faixa de menor peso ao nascimento, e mesmo assim, todas as instituições mostraram aumento das taxas de mortalidade, evidenciando que não foram as condições de nutrição da população e a prematuridade as causas das elevadas taxas encontradas.

Com a padronização indireta, na qual utilizamos também as taxas de mortalidade dos Estados Unidos acessíveis no banco de dados do
National Institute of Child Health and Human Development Neonatal Research, encontramos taxas elevadas de mortalidade nas instituições estudadas, com RPM variando entre 114,3 e 338,0 . Os resultados apontam para o fato de que a qualidade de atenção à saúde materna $\mathrm{e}$ do recém-nascido seria a importante causa dessa alteração. Para confirmar essa possibilidade, calculamos a mortalidade específica por faixa de peso ao nascimento, e apenas a Instituição 2 (menor valor de RPM) mostrou valores, coeficientes de mortalidade, iguais e até menores que da população padrão, mas com uma população estudada bastante reduzida para comparação estatística. Em todas as instituições o que chamou atenção, quando estu- 
damos a razão de mortalidade por faixa de peso (Tabela 5), foram os melhores resultados nas faixas de menores pesos ao nascimento, mostrando que estariam ocorrendo óbitos nas faixas de maiores pesos, taxas essas que são muito reduzidas em países desenvolvidos.

O mesmo método foi utilizado por Barros et al. 3 , no seu estudo na cidade de Pelotas, Rio Grande do Sul, Brasil. Seus resultados mostraram elevadas taxas de mortalidade tanto pela padronização direta como pela indireta. Esses autores concluíram que não se poderia atribuir a má performance das taxas encontradas somente às condições de nutrição da população e à diferença entre a população de Pelotas e a da Suécia (população padrão comparada). Os autores encontraram uma maior mortalidade em crianças com peso ao nascimento superior a $2.500 \mathrm{~g}$, justamente onde os países desenvolvidos já tinham alcançado melhores resultados, decorrentes de melhoria da assistência ao recém-nascido.

Nosso estudo incluiu os recém-nascidos de maior risco de morte, diferentemente do estudo de Barros et al. 3 , que analisaram todas as faixas de peso. Outra diferença observada foi a de que a população utilizada como padrão (National Institute of Child Health and Human Development Neonatal Research) apresentava na faixa de maior risco de morte a de menores pesos ao nascimento, valores muito maiores do que os encontrados no nosso estudo, o que deveria reduzir a quantidade de óbitos esperados nessas faixas de peso, o que não ocorreu.
A análise dos fatores associados à mortalidade neonatal em cada instituição, avaliado em outro estudo, mostrou que os fatores de proteção como: visitas ao pré-natal e o uso de corticosteróide ante-natal apresentaram diferenças percentuais significativas entre as instituições ( $\mathrm{p}<0,05$ e $\mathrm{p}<0,0001$, respectivamente). A Instituição 2 foi a que apresentou o menor percentual de mortalidade, e foi aquela que mostrou os maiores percentuais de fatores de proteção. A Instituição 4 apresentou os menores valores dos fatores de proteção e o segundo maior valor de RPM. A Instituição 1 embora apresentasse o segundo melhor percentual dos fatores de proteção, apresentou uma elevada quantidade de mortes de recém-nascidos, por asfixia perinatal, no primeiro dia de vida e principalmente em crianças com peso $\geq 1.000 \mathrm{~g}$.

Concluímos que ainda estamos muito distante de um sistema de saúde pública efetivo e compromissado com a melhoria no atendimento perinatal. A divulgação e o debate de vários estudos, tanto em periódicos como em congressos médicos, mostraram que mudanças no sistema atual já poderiam ter sido tomadas e os problemas analisados por Barros et al. 3 , na cidade de Pelotas, há vinte anos, não deveriam acontecer atualmente no Município do Rio de Janeiro, com taxas de mortalidade elevadas devido a uma assistência perinatal deficiente, principalmente nas faixas de maior peso ao nascimento.

\section{Resumo}

Foi realizada a comparação das taxas de mortalidade neonatal em quatro maternidades do Município do Rio de Janeiro, Brasil. A população estudada foi de recém-nascidos com peso inferior a $1.500 \mathrm{~g}$. O instrumento utilizado foi um questionário com dados informados pela mãe e o prontuário médico. Foram calculados, para cada instituição, as Razões Padronizadas de Mortalidade (RPM) com o método direto e indireto, tendo como padrão a distribuição por peso do National Institute of Child Health and Human Development Neonatal Research. A amostra final apresentou 487 recém-nascidos. As padronizações pelo método direto e indireto mostraram elevadas taxas de mortalidade em todas as instituições; as que apresentaram a maior quantidade de recém-nascidos nas faixas com os menores pesos, foram aquelas que mostraram os menores valores de RPM. A menor razão de mortalidade por faixa de peso foi encontrada na faixa de peso entre 500 e 749g. Os resultados da RPM estão inversamente associados ao quantitativo populacional de recém-nascidos nas faixas com os menores pesos. Os coeficientes de mortalidade mostraram taxas altas, principalmente nas faixas de peso mais elevados. Os resultados apontam para uma qualidade deficiente na atenção perinatal.

Mortalidade Neonatal; Recém-nascido de Muito Baixo Peso; Maternidades 


\section{Colaboradores}

J. L. M. B. Duarte colaborou com a revisão de literatura, coleta de dados, análise dos resultados e redação do artigo final. G. A. S. Mendonça contribuiu na análise dos resultados e redação do artigo final.

\section{Referências}

1. Mallett R, Knox EG. Standardized perinatal mortality ratios: techinique, utility and interpretation. Community Med 1979; 17:6-13.

2. Wilcox AJ, Russell IT. Perinatal mortality: standardizing for birthweight is biased. Am J Epidemiol 1983; 118:857-64.

3. Barros FC, Victora CG, Granzoto JA, Vaughan JP, Lemos Junior AV. Saúde perinatal em Pelotas, RS, Brasil. Fatores sociais e biológicos. Rev Saúde Pública 1984; 18:301-12.

4. Guyer B, Hoyert DL, Martin JA, Ventura SJ, MacDorman MF, Strobino DM. Annual summary of vital statistics - 1998. Pediatrics 1999; 104:1229-46.

5. Essen B, Bodker B, Sjoberg NO, Langhoff-Roos J, Greisen G, Gudmundsson S, et al. Are some perinatal deaths in immigrant groups linked to suboptimal perinatal care services? BJOG 2002; 109: 677-82.

6. Sankaran K, Chien LY, Walker R, Seshia M, Ohlsson A. Variations in mortality rates among Canadian neonatal intensive care units. CMAJ 2002; 166:173-8.

7. Rosenberg KD, Desai RA, Na Y, Kan J, Schwartz L. The effect of surfactant on birthweight-specific neonatal mortality rate, New York City. Ann Epidemiol 2001; 11:337-41.

8. Udani S. Feeding in the PICU. Indian J Pediatr 2001; 68:333-7.
9. Shankaran S, Fanaroff AA, Wright LL, Stevenson DK, Donovan EF, Ehrenkranz RA, et al. Risk factors for early death among extremely low-birthweight infants. Am J Obstet Gynecol 2002; 186: 796-802.

10. Horbar JD. The Vermont-Oxford Neonatal Network: integrating research and clinical practice to improve the quality of medical care. Semin Perinatol 1995; 19:124-31.

11. Hein HA, Lofgren MA. The changing pattern of neonatal mortality in a regionalized system of perinatal care: a current update. Pediatrics 1999; 104 (5 Pt 1):1064-9.

12. Lemons JA, Bauer CR, Oh W, Korones SB, Papile LA, Stoll BJ, et al. Very low birth weight outcomes of the National Institute of Child health and human development neonatal research network, January 1995 through December 1996. Pediatrics 2001; 107:E1.

13. Guyer B, Wallach LA, Rosen SL. Birth-weightstandardized neonatal mortality rates and the prevention of low birth weight: how does Massachusetts compare with Sweden? N Engl J Med 1982; 306:1230-3.

14. Wilcox AJ, Russell IT. Birthweight and perinatal mortality: III. Towards a new method of analysis. Int J Epidemiol 1986; 15:188-96.

Recebido em 14/Set/2004

Versão final reapresentada em 08/Abr/2005

Aprovado em 25/Abr/2005 